\title{
Luminescence of the surface nonbridging oxygen hole center in silica: Spectral and decay properties
}

\author{
L. Vaccaro, ${ }^{1,2}$ M. Cannas, ${ }^{1}$ V. Radzig, ${ }^{3}$ and R. Boscaino ${ }^{1}$ \\ ${ }^{1}$ Dipartimento di Scienze Fisiche ed Astronomiche, Università di Palermo, Via Archirafi 36, 90123 Palermo, Italy \\ ${ }^{2}$ Istituto di Biofisica CNR, Area della Ricerca di Palermo, Via Ugo La Malfa 153, 90146 Palermo, Italy \\ ${ }^{3}$ N.N. Semenov Institute of Chemical Physics, Russian Academy of Sciences, Moscow, 117977 Russia
}

(Received 9 May 2008; revised manuscript received 16 July 2008; published 18 August 2008)

\begin{abstract}
We investigated the red luminescence in a porous film of silica nanoparticles, originating from surface nonbridging oxygen hole centers. The excitation spectrum was measured from 1.8 to $8.0 \mathrm{eV}$ by a tunable laser system and a synchrotron radiation source; this spectrum evidences a peak at $2.0 \mathrm{eV}$, nearly overlapping with the emission, and an ultraviolet broadband with peaks at 4.8 and $6.0 \mathrm{eV}$. The emission is characterized by a spectrum with two subbands split by $0.07 \mathrm{eV}$, its decay occurs with lifetime longer than $30 \mu$ s and undergoes a thermal quenching by a factor $\sim 2$ with increasing temperature from 10 to $290 \mathrm{~K}$. The optical characteristics of surface and bulk centers are discussed on the basis of the reported experimental results and quantum chemical calculations.
\end{abstract}

DOI: 10.1103/PhysRevB.78.075421

PACS number(s): 73.20.Hb, 78.47.Cd, 78.55.Mb, 78.68. $+\mathrm{m}$

\section{INTRODUCTION}

Properties of silica surfaces have recently attracted attention in connection with the study of nanometer sized particles, characterized by a large specific surface area. ${ }^{1-6}$ In particular, a wide range of phenomena is strongly conditioned by the presence of surface point defects; for instance, they can act as electron traps ${ }^{7}$ or emitting centers, ${ }^{8,9}$ potentially relevant for application in electronics and optics of nanodevices. Basically, the advantage of stabilizing specific defects at a surface by controlled thermochemical processes is crucial to better understand their structural and electronic properties and those of the bulk counterpart. ${ }^{8,9}$

One of most common defects at a surface and in the bulk of silica is the oxygen dangling bond or nonbridging oxygen hole center (NBOHC) whose structure is denoted by $\equiv \mathrm{Si}-\mathrm{O}$, where $(\equiv)$ stands for bonds with three oxygen atoms and $\left(\mathbf{)}\right.$ indicates an unpaired electron. ${ }^{10,11}$ The spectroscopic properties of this center have been studied extensively in bulk systems, where it is induced by irradiation (UV, $\gamma$, and $\beta$ rays) and can be revealed both by electron paramagnetic resonance (EPR) and by optical experiments. ${ }^{12-14}$ While EPR studies have primarily had a basic importance to clarify the electronic structure of the defect in its ground state providing firm evidence that the hole is localized in a single nonbonding $2 p$ orbital of nonbridging oxygen (NBO), ${ }^{15}$ the optical properties have been considered for their practical importance as well. Indeed, NBOHC causes several optical-absorption (OA) bands in the visible $(2.0 \mathrm{eV}), \mathrm{UV}(4.8 \mathrm{eV})$, and vacuum-UV $(6.5 \mathrm{eV})$ range ${ }^{13,16-18}$ that are detrimental to the transmittance of silica devices such as optical fibers or laser optical elements. ${ }^{19-21}$ From a fundamental point of view, the most important optical feature is the photoluminescence (PL) band around 1.9 $\mathrm{eV}$ that is excited in all absorption bands and has provided the measure of spectroscopic parameters associated with the electronic transitions of the bulk NBOHC, such as the lifetime $(\sim 10 \mu \mathrm{s})$ or the strength of the phonon coupling to the stretching mode of NBO $(\sim 0.06) .{ }^{12,22}$
On the other hand, the study of the surface NBOHC has had a somewhat minor role compared to the bulk defect, in so much that its identification takes advantage of the similarity of their optical properties. The main available results are: (i) the indirect measure of the OA spectrum of powdered silica, using the diffuse reflectance technique, that shows two bands around 2.0 and $4.8 \mathrm{eV}$ (Ref. ${ }^{23}$ ); and (ii) the observation of an emission around $1.9 \mathrm{eV}$ under laser excitation within these absorption bands. ${ }^{4,5,11,24}$ The poor knowledge of the optical properties of the surface $\mathrm{NBOHC}$ is the major obstacle to the thorough understanding of the electronic structure of the excited states, an issue also discussed by computational works. ${ }^{6,9}$ In this work we, therefore, report a comprehensive study of the luminescence properties of the surface-NBOHC in a porous film of silica nanoparticles. To this aim, our experimental approach consists of the combined use of different excitation sources, a tunable laser system in the visible and UV and synchrotron radiation in the UV and vacuum UV, and time resolved detection. Moreover, the control of thermochemical processes leading to the stabilization of the NBOHC at a silica surface allows us to investigate the spectroscopic properties of a defect whose structure and symmetry are known a priori. This advantage is crucial to the discussion of our results and compare them with previous works on this issue.

\section{EXPERIMENT METHODS}

The experimental data reported here were measured on a porous film sample obtained by pressing a highly dispersed Aerosil ${ }^{\circledR}-300$, a hydrophilic fumed silica with an average particle size of $7 \mathrm{~nm}$, a pore size of 3-6 nm, and a specific surface of $\sim 10^{6} \mathrm{~cm}^{2} / \mathrm{g}$. NBOHCs were stabilized at the surface by a multistep thermochemical method starting from hydroxylated surfaces: (i) reaction of the $\equiv \mathrm{Si}-\mathrm{OH}$ group with methanol vapor $\left(\mathrm{CH}_{3} \mathrm{OH}\right)$ at $T=700 \mathrm{~K}$ and pressure of $120 \mathrm{mbar}$, leading to the generation of methoxy groups $\equiv \mathrm{Si}-\mathrm{OCH}_{3}$; (ii) high-temperature $(T>1000 \mathrm{~K})$ pyrolysis $\equiv \mathrm{Si}-\mathrm{OCH}_{3} \rightarrow \equiv \mathrm{Si}-\mathrm{H}+\mathrm{O}=\mathrm{CH}_{2}$; (iii) subsequent pyrolysis of 
$\equiv \mathrm{Si}-\mathrm{H}$ that causes the generation of surface $E$ ' centers ( $\equiv \mathrm{SiO}$ ); and (iv) treatment in $\mathrm{N}_{2} \mathrm{O}$ atmosphere at $T$ $>750 \mathrm{~K}$ accompanied by oxygen chemisorptions at the $E^{\prime}$ center site that produces the surface NBOHC. The intermediate products of the above reactions were checked by infrared and EPR spectra, thus ensuring that the NBOHC investigated in the present work are located at the silica surfaces. More details on the chemical modification of the surface defects and the characterization of their infrared and EPR parameters are given in Ref. 9. Since the surface centers are highly reactive, ${ }^{9}$ the film is placed in a pure silica container with residual $\mathrm{He}$ atmosphere of 3-4 mbar. Such a container does not show any PL related to NBOHC and is characterized by a low optical absorption, $\mathrm{OD}<0.3$ (where OD stands for optical density) up to the silica energy-gap tail around 8.0 $\mathrm{eV}$ that fixes our upper limit in the excitation spectra.

PL time resolved spectra excited in the visible and UV range were performed using a pulsed laser system (VIBRANT OPOTEK) consisting of an optical parametric oscillator pumped by the third harmonic of a Nd:YAG (where YAG stands for yttrium aluminum garnet) laser (pulse width $\approx 5 \mathrm{~ns}$, repetition rate of $10 \mathrm{~Hz}$ ) and equipped with a nonlinear crystal for the second-harmonic generation. The sample was excited by the laser beam (spot size $\sim 1 \mathrm{~mm}^{2}$ ) in a so-called $45^{\circ}$ backscattering geometry. The emitted light was spectrally resolved by a monochromator equipped with a 300 grooves/mm grating blazed at $500 \mathrm{~nm}$, and then acquired by an intensified charge coupled device (CCD) camera driven by a delay generator (PI-MAX Princeton Instruments) that sets the gate width $W_{T}$ and the delay time $T_{D}$ after the laser pulse. All the emission spectra were detected with a bandwidth of $10 \mathrm{~nm}$ and were corrected for the monochromator dispersion; their intensity was also normalized to the laser-pulse energy. The photoluminescence excitation (PLE) spectra were measured manually point by point by tuning the laser and recording the integrated PL intensity.

To extend the excitation range up to vacuum UV, PL and PLE spectra were measured under pulsed synchrotron radiation $(\mathrm{SR}$ ) (pulse width $\approx 130 \mathrm{ps}$, interpulse $\approx 192 \mathrm{~ns}$, photon energy ranging from 4 to $8 \mathrm{eV}$, and spectral width of $0.3 \mathrm{~nm}$ ) at the SUPERLUMI station on the I-beamline of HASYLAB at DESY (Hamburg). The emitted light was spectrally dispersed by a 300 grooves $/ \mathrm{mm}$ grating blazed at $500 \mathrm{~nm}$ and acquired by a liquid nitrogen cooled CCD camera (1100 Princeton Instruments) for PL spectra or by a photomultiplier (Hamamatsu R2059) with $W_{T}=100 \mathrm{~ns}$ and $T_{D}=103 \mathrm{~ns}$ from the SR pulse for PLE spectra. The PL spectra were detected with an emission bandwidth of $10 \mathrm{~nm}$ and were corrected for the monochromator dispersion. The PLE spectra were detected with an emission bandwidth of $20 \mathrm{~nm}$ and were corrected both for the spectral efficiency of the exciting light, using a sodium salicylate sample as a reference, and for the light transmission of the silica container.

Both experimental stations were equipped with continuous flow helium cryostats, and the spectra were acquired at various temperatures in the range 10-290 K.

\section{RESULTS}

Figure 1 shows the luminescence properties of the surface NBOHC derived by time resolved spectra detected with

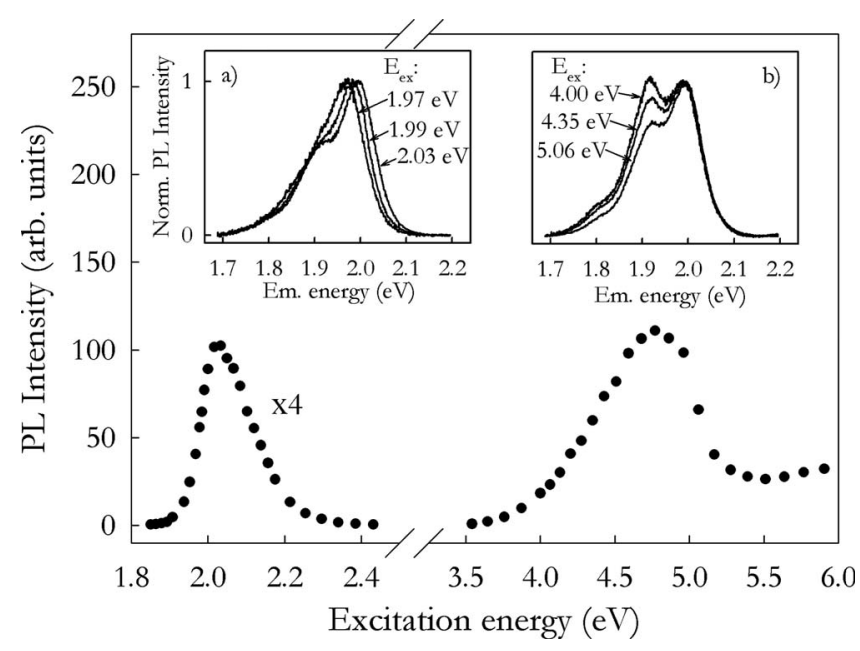

FIG. 1. Excitation spectrum of the surface NBOHC luminescence measured under laser radiation and detected with $W_{T}=1 \mu \mathrm{s}$ and $T_{D}=1 \mu \mathrm{s}$. The insets show the normalized PL spectra measured at different excitation energies in the (a) visible and (b) UV range.

$W_{T}=1 \mu \mathrm{s}$ and $T_{D}=1 \mu \mathrm{s}$, under laser excitation $E_{\text {ex }}$ ranging from 1.85 to $2.43 \mathrm{eV}$ and from 3.54 to $5.90 \mathrm{eV}$; the pulse energy being fixed to be 0.5 and $0.2 \mathrm{~mJ}$ in the two ranges, respectively. The PL spectrum is characterized by a red band whose shape depends on $E_{\text {ex }}$. As evidenced in inset (a), the increase in $E_{\mathrm{ex}}$ in the visible range results in a shift of the PL band toward higher energies: its maximum changes from $1.97 \pm 0.01 \mathrm{eV}$ (at $E_{\mathrm{ex}}=1.97 \mathrm{eV}$ ) to $2.00 \pm 0.01 \mathrm{eV}$ (at $E_{\mathrm{ex}}$ $=2.03 \mathrm{eV}$ ). Moreover, at $E_{\mathrm{ex}}=2.03 \mathrm{eV}$ a component at lower energies is also observed. The excitation spectrum displays an asymmetric band peaked at $2.03 \pm 0.02 \mathrm{eV}$ with full width at half maximum (FWHM) of $0.16 \pm 0.02 \mathrm{eV}$. Under UV excitation the PL spectra, reported in the inset (b), show markedly two subbands peaked at $1.92 \pm 0.01 \mathrm{eV}$ and $1.99 \pm 0.01 \mathrm{eV}$ whose amplitude ratio depends on the excitation energy. In this range, the excitation spectrum is characterized by a band peaked at $4.77 \pm 0.02 \mathrm{eV}$ and with $\mathrm{FWHM}=0.88 \pm 0.02 \mathrm{eV}$, overlapping with a component at higher energies.

Through the SR excitation, we have achieved the PLE spectrum monitored at $E_{\mathrm{em}}=1.98 \mathrm{eV}$ and reported in Fig. 2. Besides the band at $4.77 \mathrm{eV}$, already displayed in Fig. 1, it shows further components in the vacuum UV spectral range, the main being peaked at $6.05 \pm 0.02 \mathrm{eV}$. In the inset we report the normalized PL spectra acquired with $E_{\mathrm{ex}}=4.77$ and $6.05 \mathrm{eV}$, both show two subbands in the red spectral range. In addition, a PL component extending to higher energies is observed under vacuum UV excitation; a similar band, excited by Argon Fluorine laser $(6.4 \mathrm{eV})$, was reported in previous works on silica nanoparticles and was ascribed to hydrogen related species. ${ }^{5,24}$

The temperature dependence of the surface NBOHC PL is shown in Fig. 3 by time resolved spectra measured under laser excitation at $4.77 \mathrm{eV}$. Over the investigated range, 10$290 \mathrm{~K}$, the emission spectrum consists of two subbands. On increasing temperature both bands broaden and their peak values decrease; moreover their splitting slightly increases 


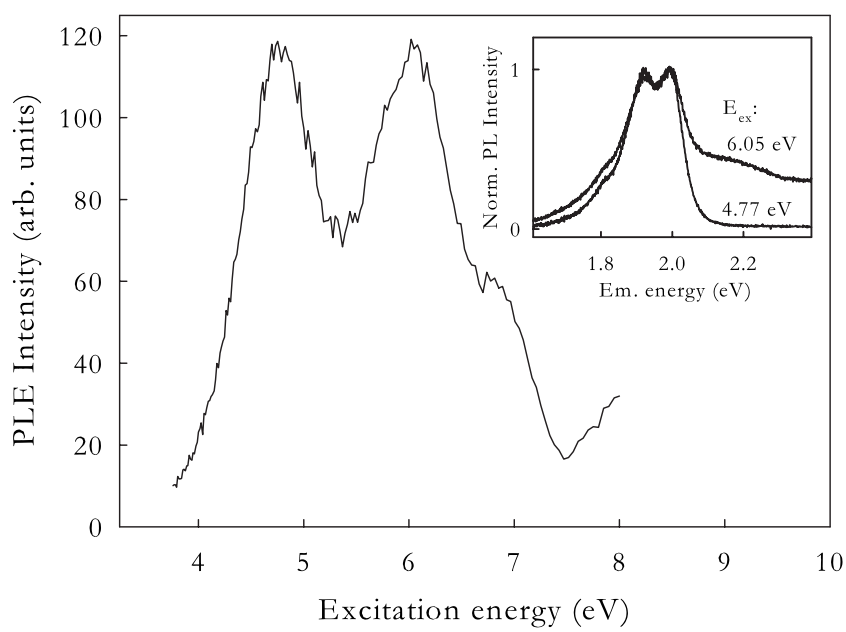

FIG. 2. Excitation spectrum monitored at $E_{\mathrm{em}}=1.98 \mathrm{eV}$ with $W_{T}=100 \mathrm{~ns}$ and $T_{D}=103 \mathrm{~ns}$ detected under synchrotron radiation ranging from 4 to $8 \mathrm{eV}$. The inset shows the normalized PL spectra measured with $E_{\mathrm{ex}}=4.77$ and $6.05 \mathrm{eV}$.

from $0.06(10 \mathrm{~K})$ to $0.07 \mathrm{eV}(290 \mathrm{~K})$. In the inset we report the integrated intensity as a function of temperature: at $T$ $<100 \mathrm{~K}$ the emission is nearly temperature independent, it decreases by a factor $\sim 2$ in the range $100<T<220 \mathrm{~K}$ and then it remains constant up to room temperature. The same trend has been measured under SR excitation in the vacuum UV range.

In Fig. 4 we report the time resolved PL spectra acquired under pulsed excitation at (a) 4.77 and (b) $2.07 \mathrm{eV}$, with $W_{T}=4 \mu \mathrm{s}$ and $T_{D}$ ranging up to $248 \mu \mathrm{s}$, when the decay of the two emission subbands is almost completed. Figure 5(a) shows the decay kinetics of the PL intensity, $I(t)$, at $E_{\mathrm{em}}$ $=1.91$ and $1.99 \mathrm{eV}$. As evident from the semilogarithmic scale, the PL decay curves deviate from a single exponential law. This behavior is common to other defects in silica, such as oxygen deficient centers, ${ }^{25,26}$ and is consistent with a mul-

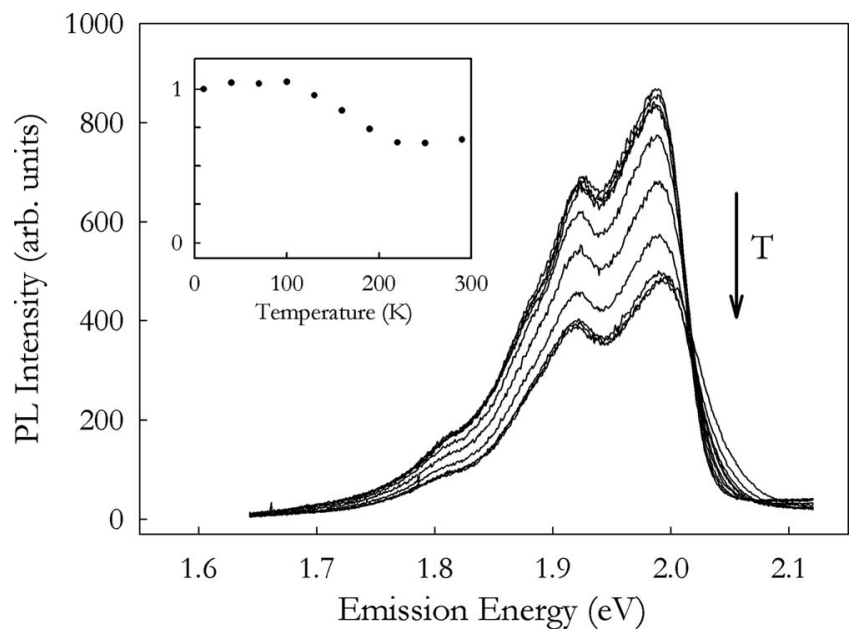

FIG. 3. PL spectra measured at different temperatures (10-290 $\mathrm{K})$ under laser excitation at $4.77 \mathrm{eV}$. The arrow indicates the changes induced in the spectra with increasing temperature. The inset shows the temperature dependence of the PL integrated intensity.
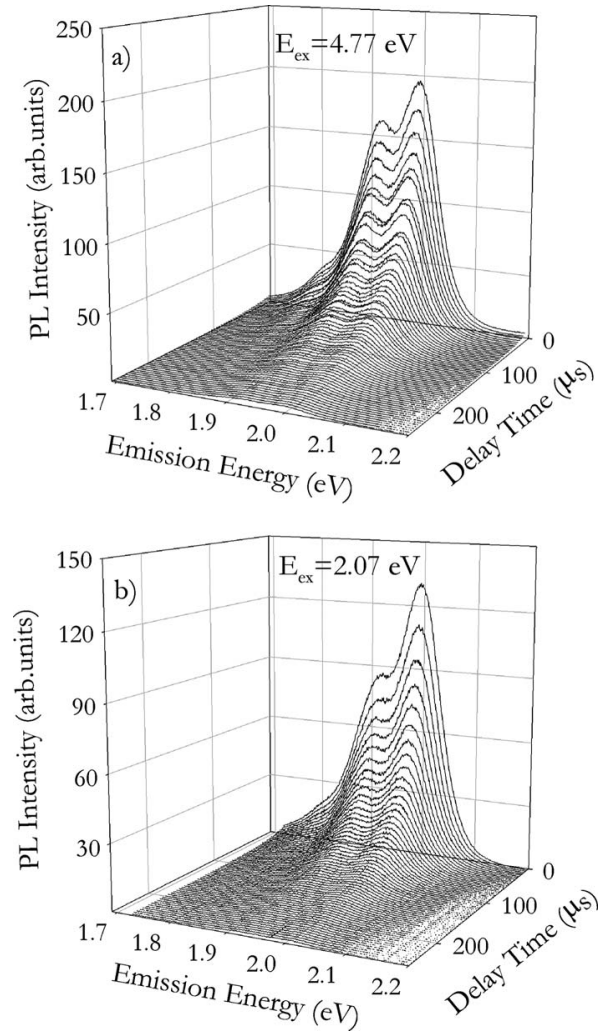

FIG. 4. Time resolved PL spectra measured under laser excitation at (a) 2.07 and (b) $4.77 \mathrm{eV}$.

tiexponential decay with decay constants inhomogeneously distributed. The quantitative analysis was therefore carried out with the stretched exponential law,

$$
I(t) \propto \exp \left[-(t / \tau)^{\gamma}\right],
$$

where $\tau$ is the decay time or lifetime and $\gamma$ is the stretching parameter that measures the deviation from a single exponential law. The results of the fitting procedure are reported in Table I. Finally, in Fig. 5(b) we show the decay kinetics of the PL band at $E_{\mathrm{em}}=1.99 \mathrm{eV}$ measured under excitation at $4.77 \mathrm{eV}$ at different temperatures. The deviation from a single exponential law is maintained in the investigated temperature range and the lifetime slightly increases from $\tau$ $=41.2 \pm 0.5 \mu \mathrm{s}$ at $T=300 \mathrm{~K}$ to $\tau=52.0 \pm 0.5 \mu \mathrm{s}$ at $T$ $=10 \mathrm{~K}$.

\section{DISCUSSION}

The present data show that the optical properties of the surface NBOHC are characterized by an emission around 1.9-2.0 eV whose excitation spectrum consists of a band at $2.0 \mathrm{eV}$ and a broadband extending from UV to vacuum UV with significant peaks at 4.8 and at $6.0 \mathrm{eV}$; these excitation bands allow us to identify the spectral range of the absorption transitions. Previous studies have evidenced that the bulk NBOHC is also characterized by an emission peaked at $1.9 \mathrm{eV}(\mathrm{FWHM} \approx 0.17 \mathrm{eV})$ having an excitation spectrum with several bands peaked at $2.0(\mathrm{FWHM} \approx 0.17 \mathrm{eV}), 4.8$ $(\mathrm{FWHM} \approx 1.0 \mathrm{eV})$, and $6.4 \mathrm{eV}(\mathrm{FWHM} \approx 1.7 \mathrm{eV}) .{ }^{11,12,14}$ 


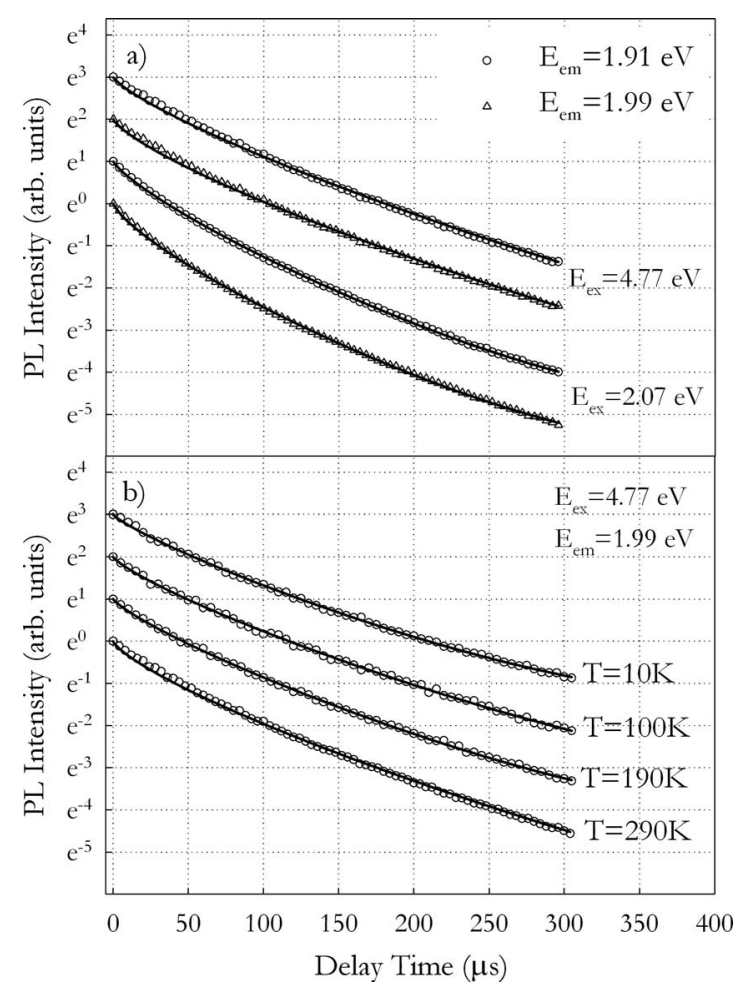

FIG. 5. Decay curves detected (a) at room temperature at $E_{\mathrm{em}}$ $=1.91$ and $1.99 \mathrm{eV}$ under laser excitation at $E_{\mathrm{ex}}=4.77$ and $2.07 \mathrm{eV}$ and (b) at different temperatures at $E_{\mathrm{em}}=1.91 \mathrm{eV}$ under $E_{\mathrm{ex}}$ $=4.77 \mathrm{eV}$. For viewing purposes, the initial values of the decay curves are arbitrarily scaled. Full lines plot the best fit curves of Eq. (1).

Regardless of the environment, the spectral features of NBOHC can be accounted for by an energy-level scheme where two different pathways of excitation/emission can be distinguished. The first includes the cycle $2.0 \mathrm{eV}$ (excitation)/1.9 eV (emission) occurring between two electronic states whose small Stokes shift is consistent with a very weak electron-phonon coupling. ${ }^{12}$ We can calculate the oscillator strength of the $2.0 \mathrm{eV}$ absorption band using the luminescence lifetime at low temperature $(\tau \approx 50 \mu \mathrm{s})$ and the Einstein coefficient relation, ${ }^{12} f \approx 6 \times 10^{-5}$. The second cycle, UV and vacuum UV (excitation)/1.9 eV (emission), involves additional electronic states and the large Stokes shift is due to nonradiative electronic relaxations.

The similarity between the energy-level schemes of bulk and surface NBOHC is consistent with the transitions between the same molecular orbitals; however some spectro-

TABLE I. Decay time and stretching parameter of the surfaceNBOHC luminescence extracted using the best fit Eq. (1).

\begin{tabular}{llcc}
\hline \hline & & $\begin{array}{c}\tau \\
(\mu \mathrm{s})\end{array}$ & $\gamma$ \\
\hline$E_{\mathrm{ex}}=4.77 \mathrm{eV}$ & $E_{\mathrm{em}}=1.92 \mathrm{eV}$ & $44.3 \pm 0.5$ & $0.78 \pm 0.02$ \\
& $E_{\mathrm{em}}=1.99 \mathrm{eV}$ & $41.2 \pm 0.5$ & $0.76 \pm 0.02$ \\
$E_{\mathrm{ex}}=2.07 \mathrm{eV}$ & $E_{\mathrm{em}}=1.92 \mathrm{eV}$ & $35.2 \pm 0.5$ & $0.79 \pm 0.02$ \\
& $E_{\mathrm{em}}=1.99 \mathrm{eV}$ & $29.9 \pm 0.5$ & $0.75 \pm 0.02$ \\
\hline \hline
\end{tabular}
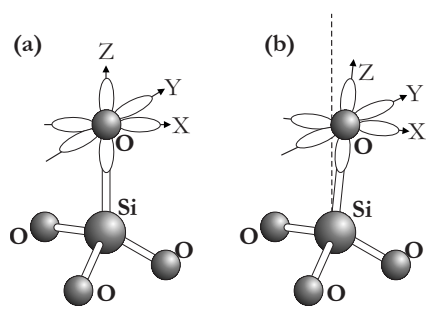

FIG. 6. Structure of the NBOHC and $p$ orbitals of the dangling oxygen in the (a) $C_{3 v}$ and (b) $C_{S}$ symmetries.

scopic properties are influenced by different environment. In the surface defect the PL spectrum is composed of two subbands, the energy splitting between them, measured under $\mathrm{UV}$ and vacuum UV excitation, is $\sim 0.07 \mathrm{eV}$ at room temperature; the PL lifetime is longer than $30 \mu$ s and its intensity is weakly temperature dependent. In contrast, in the bulk defect the PL spectrum is structureless, its decay time, measured under 2.0, 4.8, and $6.4 \mathrm{eV}$, follows a stretched exponential curve with a lifetime of $\sim 10 \mu$ s, and it has a larger dependence on temperature (a factor $\sim 10$ in the range 10 $300 \mathrm{~K})$. $^{11,17,18}$

As a whole, these features can be discussed on the basis of the geometrical and electronic properties of the NBOHC derived by the models proposed so far. As pointed out in the experimental section, the surface $\mathrm{NBOHC}$ is generated by the reaction of $\mathrm{N}_{2} \mathrm{O}$ with the surface $E^{\prime}$ center, an isolated threefold coordinated $\mathrm{Si}(\equiv \mathrm{Si})$. Then, its symmetry is expected to be $C_{3 v}$, in accordance with the structure of the surface $E^{\prime}$ coordination sphere that is known by the EPR properties: an axially symmetric $g$ tensor and nearly coincident hyperfine constants due to the interaction with the ${ }^{29} \mathrm{Si}$ atoms bonded to the three basal O. ${ }^{27}$ In Fig. 6(a) we report the structural model of the surface $\mathrm{NBOHC}$ with a $C_{3 v}$ symmetry; the $2 p$ orbitals of the dangling oxygen are also shown: $2 p_{x}$ lies in the symmetry plane and $2 p_{y}$ is orthogonal to the symmetry plane. Quantum chemical calculations have shown that, due to the Jahn-Teller effect, the symmetry of the surface NBOHC deviates from $C_{3 v}$ and becomes $C_{S}$, as sketched in Fig. 6(b), thus removing the orbital electron degeneracy of the ground state between $2 p_{x}$ and $2 p_{y}$; the calculated energy difference between these states is $\sim 0.1 \mathrm{eV} .{ }^{9}$ This result has been confirmed by further computational works that have shown that the switch of the unpaired electron from the $2 p_{x}$ to $2 p_{y}$ orbitals gives rise to a transition at $0.04-0.24 \mathrm{eV}$ with a very low oscillator strength $(f \sim 7$ $\left.\times 10^{-6}\right){ }^{6,28,29}$

With regard to the exited state from which the PL takes place, there is a general consensus that it is characterized by a lone pair in both nonbonding $2 p$ orbitals of NBO. ${ }^{6,28-33}$ According to this computational picture, the two subbands observed in the PL spectrum can be ascribed to a charge transfer leaving the unpaired electron on $2 p_{x}$ or $2 p_{y}$ orbitals, whose energy difference is measured here to be 0.06-0.07 $\mathrm{eV}$ over the temperature range $10-290 \mathrm{~K}$, thus supporting the values predicted in the former computational works, ${ }^{6,9,28,29}$ despite this is not a direct prove of the occurrence of the Jahn-Teller effect. As evidenced above, these two subbands are not observed in the PL spectra of the bulk NBOHC. A 
straightforward explanation can be derived by the different influence of the bulk and surface silica network on the PL broadening, both homogenous due to the phonon coupling ${ }^{34}$ and inhomogeneous due to the disordered silica network. The second contribution can be singled out by site-selective techniques; ${ }^{22,35}$ in the bulk NBOHC it results in a Gaussian curve with $(\mathrm{FWHM})_{\text {inh }} \sim 0.08 \mathrm{eV}$, it is large enough to prevent the spectral resolution of the two subbands. In contrast, in the surface NBOHC the observation of the two subbands agrees with a narrower inhomogeneous broadening due to a lower influence of disorder. ${ }^{36}$

The long lifetime measured in the surface NBOHC $(\tau$ $>30 \mu \mathrm{s})$ points out the forbidden character of the PL transition and then the small overlap between the filled $2 p$ orbitals of NBO that identify the excited state and that where the charge transfer terminates. The same orbitals overlap more in the bulk defect, where the lifetime is $\tau \sim 10 \mu \mathrm{s}$. This result is a clue for the different NBOHC geometry in the two networks. While at the surface, NBO interacts effectively only with the atoms of its own tetrahedron, in the bulk it also experiences the influence of neighboring atoms $(\mathrm{O}, \mathrm{Si}$, and $\mathrm{H})$ whose nature depends on the defect generation process. ${ }^{10,12}$ The bulk NBOHC has therefore a $C_{S}$ symmetry, Fig. 6(b), with a larger deviation from $C_{3 v}$ that favors the overlap described above between the orbitals involved in the PL transition. The thermal quenching of the PL due to the activation of nonradiative channels in the excitation/emission pathway is also an evidence of the weaker interaction of the surface NBOHC with its environment in comparison with the bulk. Moreover, we observe that temperature poorly affects the lifetime. This result is common to the bulk ${ }^{37}$ and points out that the nonradiative relaxation affects mainly the populating of the luminescent level.

\section{CONCLUSIONS}

The results reported here provide a valid support in understanding the luminescence properties of the surface NBOHC whose structure and symmetry are well known, thanks to the controlled chemical reactions leading to its generation in a porous film of silica nanoparticles. This advantage, not available in the bulk counterpart, plays a crucial role in the comparison of the computational models dealing with the properties of almost unperturbed defects; in particular it corroborates the previous calculations predicting the energy splitting between the $2 p$ orbitals of NBO in the ground state.

\section{ACKNOWLEDGMENTS}

The authors thank F. Messina and E. Vella for their support during the experimental session at DESY and the members of the LAMP group (www.fisica.unipa.it//amorphous) for the useful discussions. Technical assistance by G. Napoli, F. Testaino, and G. Tricomi is also acknowledged.
${ }^{1}$ K. S. Seol, Y. Ohki, H. Nishikawa, M. Takiyama, and Y. Hama, J. Appl. Phys. 80, 6444 (1996).

${ }^{2}$ M. Goldberg, H.-J. Fitting, and A. Trukhin, J. Non-Cryst. Solids 220, 69 (1997).

${ }^{3}$ Defects in $\mathrm{SiO}_{2}$ and Related Dielectrics: Science and Technology, edited by G. Pacchioni, L. Skuja, and D. Griscom (Kluwer, Dordrecht, 2000).

${ }^{4}$ Yu. D. Glinka, S.-H. Lin, and Y.-T. Chen, Phys. Rev. B 62, 4733 (2000).

${ }^{5}$ Yu. D. Glinka, S.-H. Lin, and Y.-T. Chen, Phys. Rev. B 66, 035404 (2002).

${ }^{6}$ L. Giordano, P. V. Sushko, G. Pacchioni, and A. L. Shluger, Phys. Rev. B 75, 024109 (2007).

${ }^{7}$ L. Giordano, P. V. Sushko, G. Pacchioni, and A. L. Shluger, Phys. Rev. Lett. 99, 136801 (2007).

${ }^{8}$ V. A. Radzig, Chem. Phys. Rep. 14, 1206 (1995).

${ }^{9}$ V. A. Radzig, in Defects in $\mathrm{SiO}_{2}$ and Related Dielectrics: Science and Technology, edited by G. Pacchioni, L. Skuja, and D. Griscom (Kluwer, Dordrecht, 2000).

${ }^{10}$ D. L. Griscom, J. Ceram. Soc. Jpn. 99, 923 (1991).

${ }^{11}$ L. Skuja, J. Non-Cryst. Solids 179, 51 (1994).

${ }^{12}$ L. Skuja, in Defects in $\mathrm{SiO}_{2}$ and Related Dielectrics: Science and Technology, edited by G. Pacchioni, L. Skuja, and D. Griscom (Kluwer, Dordrecht, 2000).

${ }^{13}$ K. Kajihara, Y. Ikuta, M. Hirano, and H. Hosono, Appl. Phys. Lett. 81, 3164 (2002).

${ }^{14}$ M. Cannas, L. Vaccaro, and B. Boizot, J. Non-Cryst. Solids 352,
203 (2006).

${ }^{15}$ M. Stapelbroek, D. L. Griscom, E. J. Friebele, and G. H. Sigel, Jr., J. Non-Cryst. Solids 32, 313 (1979).

${ }^{16}$ L. Skuja, K. Tanimura, and N. Itoh, J. Appl. Phys. 80, 3518 (1996).

${ }^{17}$ H. Hosono, K. Kajihara, T. Suzuki, Y. Ikuta, L. Skuja, and M. Hirano, Solid State Commun. 122, 117 (2002).

${ }^{18}$ M. Cannas and F. M. Gelardi, Phys. Rev. B 69, 153201 (2004).

${ }^{19}$ M. A. Stevens-Kalceff, A. Stesmans, and J. Wong, Appl. Phys. Lett. 80, 758 (2002).

${ }^{20}$ S. O. Kucheyev and S. G. Demos, Appl. Phys. Lett. 82, 3230 (2003).

${ }^{21}$ S. Girard, J.-P. Meunier, Y. Ouerdane, A. Boukenter, B. Vincent, and A. Boudrioua, Appl. Phys. Lett. 84, 4215 (2004).

${ }^{22}$ L. Skuja, T. Suzuki, and K. Tanimura, Phys. Rev. B 52, 15208 (1995).

${ }^{23}$ A. Bobyshev and V. Radzig, Fiz. Khim. Stekla 14, 501 (1988).

${ }^{24}$ Yu. D. Glinka, S.-H. Lin, and Y.-T. Chen, Appl. Phys. Lett. 75, 778 (1999).

${ }^{25}$ K. S. Seol, M. Fujimaki, Y. Ohki, and H. Nishikawa, Phys. Rev. B 59, 1590 (1999).

${ }^{26}$ S. Agnello, R. Boscaino, M. Cannas, A. Cannizzo, F. M. Gelardi, S. Grandi, and M. Leone, Phys. Rev. B 68, 165201 (2003).

${ }^{27}$ V. A. Radzig, Chem. Phys. Rep. 19, 469 (2001).

${ }^{28}$ Z. Hajnal, P. Deák, Th. Köhler, R. Kaschner, and Th. Frauenheim, Solid State Commun. 108, 93 (1998).

${ }^{29}$ C. Sousa, C. De Graaf, and G. Pacchioni, J. Chem. Phys. 114, 
6259 (2001)

${ }^{30}$ L. Skuja, Solid State Commun. 84, 613 (1992).

${ }^{31}$ T. Suzuki, L. Skuja, K. Kajihara, M. Hirano, T. Kamiya, and H. Hosono, Phys. Rev. Lett. 90, 186404 (2003).

${ }^{32}$ T. Bakos, S. N. Rashkeev, and S. T. Pantelides, Phys. Rev. Lett. 91, 226402 (2003).

${ }^{33}$ T. Bakos, S. N. Rashkeev, and S. T. Pantelides, Phys. Rev. B 70, 075203 (2004).

${ }^{34}$ L. Vaccaro, M. Cannas, and R. Boscaino, J. Lumin. 128, 1132
(2008).

${ }^{35}$ L. Vaccaro, M. Cannas, and R. Boscaino, Solid State Commun. 146, 148 (2008).

${ }^{36}$ Site-selective PL measurements on the film sample investigated in the present paper have evidenced that the energy distribution of the zero-phonon line amplitude is peaked around $1.99 \mathrm{eV}$ with FWHM of $0.04 \mathrm{eV}$, unpublished data.

${ }^{37}$ J. H. Stathis and M. A. Kastner, Phys. Rev. B 35, 2972 (1987). 